\title{
XYLOGLUCAN CONJUGATED FUNCTIONALIZED GRAPHENE OXIDE AS A NANO CARRIER SYSTEM FOR pH RESPONSIVE TARGETED DRUG DELIVERY OF FUCOIDAN
}

\author{
K. SONIA ${ }^{*}$, D. RAJESH ${ }^{2}$, S. ARUNA SHARMILI ${ }^{3}$, K. S. MEENA ${ }^{4 *}$
}

${ }^{1}$ Department of Chemistry, Queen Mary's College (Autonomous), Mylapore, Chennai, Tamilnadu, 600004, India, ${ }^{2}$ Department of Microbiology, Chennai National Arts and Science College, Avadi, Chennai, Tamil Nadu, 600054, 3Department of Biotechnology, Stella Maris College (Autonomous), Chennai, Tamil Nadu, 600086, India, ${ }^{4}$ Alagappa Government Arts College, Karaikudi, Sivaganga Dist; Tamilnadu, 630003, India *Email: meenamohan90@gmail.com

Received: 26 Jun 2021, Revised and Accepted: 31 Jul 2021

\section{ABSTRACT}

Objective: Marine polysaccharides are materializing in the field of biomedicine owing to its promising properties, including high biocompatibility, excellent biodegradability, nontoxic nature, abundance and low cost. Fucoidan (FU), a sulphated marine polysaccharide extracted from brown seaweed, shows a promising application prospect as an anticancer model drug. In order to enhance the stability, biocompatibility and drug loading capacity, xyloglucan was chosen as a targeting ligand, conjugated onto the surface of chitosan functionalized graphene oxide for targeted delivery of fucoidan.

Methods: Firstly, Graphene oxide (GO) was prepared by modified Hummer's method and functionalized with chitosan (CS) via amidation process, further conjugated with xyloglucan (XG). The resulting conjugate, GO-CS-XG, was used to deliver fucoidan through a nanocarrier drug delivery method. The developed GO-CS-XG-FU nanosystem was analyzed for its physiochemical characterization, morphology, hemolytic activity, antiinflammatory and anticancer activity.

Results: The FU loading efficiency and capacity were $75.7 \%$ and $83.4 \%$, respectively. XG ligands on the nanoparticle may lead the nanoparticles to actively target cancer cells. Hemolytic activity of the FU-loaded GO-CS-XG nanosystem shows negligible activity, thus making it a potential candidate for biomedical applications. In vitro drug release analysis of FU from GO-CS-XG was lesser at physiological pH but under acidic conditions, it was significantly increased. Results of in vitro cell viability studies indicate that the efficiency of fucoidan was improved upon conjugation with the nanosystem (GO-CS-XG) against human histiocytic lymphoma (U 937) cell line.

Conclusion: As a result, we propose a new multifunctional graphene-based targeted platform by using xyloglucan polysaccharide as targeting nano material for pH-responsive anticancer drug delivery with high efficacy.

Keywords: Graphene oxide, Chitosan, Xyloglucan, Fucoidan, pH-responsive, Hemocompatibilty

(C) 2021 The Authors. Published by Innovare Academic Sciences Pvt Ltd. This is an open access article under the CC BY license (https://creativecommons.org/licenses/by/4.0/) DOI: https://dx.doi.org/10.22159/ijap.2021v13i5.42545. Journal homepage: https://innovareacademics.in/journals/index.php/ijap

\section{INTRODUCTION}

Drug conjugation to macromolecular carriers has emerged as a viable technique in the field of drug delivery systems in recent years. Because of their biocompatibility, biodegradability, non-toxicity, and simplicity of availability from natural resources, polysaccharides have attracted increased interest in drug delivery methods. Polysaccharides-based drug delivery systems are fascinating due to their numerous advantages such as prolonged half-life, solubility of drugs, improved pharmacokinetics, increased drug accumulation in tumor tissues via enhanced permeability and retention effect [1-3].

The most salient derivative of graphene is graphene oxide (G0) which consists of a wide range of oxygen-containing functional groups such as carboxyl, hydroxyl and epoxy groups on its surface. Due to its enormous surface area, $\pi$-stacking, and hydrophobic interactions, GO has attracted interest as a suitable drug carrier [4]. However, GO's dispersion in biological solution is limited, resulting in the development of aggregates, limiting its use in biomedical applications. Therefore, functionalization of GO is necessary in order to build an efficient nanocarrier system to improve its stability, biocompatibility and more prominently, interaction with the target molecules to release the drugs in a controlled way at the specific target site [5].

For example, chitosan (CS) is widely used in the study of GO-based targeted drug delivery systems due to its prominent properties such as high biocompatibility, biodegradability, bacteriostatic action, nontoxic and abundance in nature. Moreover, the positive nature of CS with free amino groups and hydroxyl groups on the polymeric chains made more beneficial for surface adhesion and enables crosslinking with the carboxyl groups of GO on its surface [6]. Thus chitosan-functionalized graphene oxide (GO-CS) nanocarrier enables good biocompatibility and bioadhesive property, which enables the prolonged retention onto the targeted substrate.
Xyloglucan (XG) is also a natural polysaccharide obtained from tamarind seeds. It is composed of a $(1 \rightarrow 4)$-linked $\beta$-D-glucan backbone chain at 0-6 position of D-glucopyranosyl residues with $\alpha$ D-xylopyranose or with 2-0- $\beta$-D-galactopyranosyl- $\alpha$-D-xylopyranose [7]. XG has gained considerable attention to carry out the work because of its striking properties, which include high viscosity, broad $\mathrm{pH}$ tolerance, non-carcinogenicity, biocompatibility, mucoadhesivity in situ gelation and high drug loading capacity [8]. Due to these versatile properties, $X G$ is considered to be an attractive and functional polymer which is particularly suitable as a nanocarrier for drug delivery systems [9]. However, until now, no targeted drug delivery systems based on xyloglucan as targeting ligand have been synthesized. Therefore, we made XG a polysaccharide of choice as a targeting ligand in the chitosan functionalized GO drug delivery systems.

Sulphated polysaccharides extracted from marine algae are excellent drug candidates for pharmaceutical applications. Over the past years, many researchers have investigated on sulphated polysaccharides-based nanomaterials for biomedical application such as antimicrobial activity, drug delivery, gene delivery, tissue engineering, wound dressing and cancer therapy $[10,11]$.

Fucoidan (FU), a naturally occuring sulphated polysaccharides mainly comprised of fucose residues and sulphate groups extracted from marine brown seaweed Sargassum wightii. It has various biological properties such as antiviral, anticoagulant, anti-tumor, anti-inflammatory, antioxidant, immunomodulating and anticoagulant [12, 13]. A vast number of studies have been conducted on the use of fucoidan as a reducing and stabilizing agent in the biosynthesis of metal nanoparticles. However, the use of FU as a model anticancer drug for GO nano carrier-based targeted drug delivery system still remain undetected. 
The purpose of the study was to functionalize the GO surface with CS via an amide bond. A novel multifunctional platform was designed by conjugating XG onto the surface of GO-CS. GO-CS-XG conjugate system was structurally and morphologically analyzed by UV-Visible, FT-IR, TEM, SEM and zeta potential. FU was used as an anti-tumor model drug to evaluate drug loading and release properties, as well as the effect of $\mathrm{pH}$. In addition, the anticancer activity of $\mathrm{pH}$-responsive $\mathrm{FU}$ loaded drug delivery systems was evaluated by MTT assay.

\section{MATERIALS AND METHODS}

\section{Materials}

Graphite powder, $30 \%$ hydrogen peroxide (H202), Nhydroxysuccinimide (NHS), 1-(3-Dimethylaminopropyl)-3-ethyl carbodiimide hydrochloride (EDC), sulphuric acid (H2SO4), hydrochloric acid $(\mathrm{HCl})$ and ethanol were obtained from Sisco Research Laboratories Pvt Ltd, (Mumbai, India). Chitosan (CS) was purchased from Marine Hydrocolloid (Kerala), Xyloglucan extracted from Tamarind seed powder was obtained as a gift sample from Indra Agrotech Private Ltd (Maharashtra, India). Brown seaweed Sargassum wightii was collected from Mandapam in the Gulf of Mannar. Fetal bovine serum (FBS), DMEM medium, antibiotics were purchased from HiMedia, India. A human histiocytic lymphoma (U 937) cell was obtained from National Centre for Cell Science (NCCS), Pune, India. All other reagents used in this research were of analytical quality.

\section{Collection of seaweed material}

The brown seaweed sargassum wightii was collected from marine water seashore was identified and authenticated by Dr. M. Ganesan, Scientist of Marine Algal Research Station, CSMCRI (Central Salt and Marine chemicals Research Institute) Mandapam Camp, Tamil Nadu, India. The seaweed was washed thoroughly with tap water and distilled water to remove extraneous materials and shade-dried for $5 \mathrm{~d}$ and oven-dried at $60{ }^{\circ} \mathrm{C}$ until a constant weight was obtained, then was grind into a fine powder using the electric mixer and stored at $4{ }^{\circ} \mathrm{C}$ for future use.

\section{Extraction of fucoidan}

Extraction of fucoidan from brown seaweed Sargassum wightii was done by the accompanying methods $[14,15]$ with some modification. Algal powder was mixed with $100 \mathrm{ml}$ of $85 \%$ ethanol and stirred at room temperature for $12 \mathrm{~h}$. In order to remove pigments, proteins and lipids, then centrifuged at $970 \mathrm{~g}$ for $10 \mathrm{~min}$. The resultant residue was then washed with acetone and left overnight to dry at room temperature. 10 gm of the algal sediment was then collected and mixed with distilled water and placed at 70 ${ }^{\circ} \mathrm{C}$ on a hot plate with constant stirring for $1 \mathrm{~h}$. The solutions were then centrifuged at $3800 \mathrm{~g}$ for $10 \mathrm{~min}$ to this $1 \% \mathrm{CaCl}_{2}$ was added in order to precipitate alginates. The solutions were then centrifuged at $3273 \mathrm{~g}$ for $10 \mathrm{~min}$ to remove unwanted impurities. The supernatant was collected and $95 \%$ ethanol was added until a final concentration of $70 \%$ was reached and stored at $4{ }^{\circ} \mathrm{C}$ overnight in order to obtain ethanol-precipitated fucoidan, was then recovered by centrifugation at $3800 \mathrm{~g}$ for $30 \mathrm{~min}$, and the precipitated fucoidan was lyophilized and stored for further analysis.

\section{Synthesis of graphene oxide (GO)}

Graphene oxide (GO) was synthesized from graphite powder according to a modified Hummer's method $[16,17]$. Briefly, graphite powder was poured into the cold solution of concentrated $\mathrm{H}_{2} \mathrm{So}_{4}$ and $\mathrm{NaNO}_{3}$. Then, $\mathrm{KMnO}_{4}$ was gradually added to this solution upon continuous stirring under ice bath medium maintaining a temperature of $10-15{ }^{\circ} \mathrm{C}$. The reaction mixture was then stirred at $40{ }^{\circ} \mathrm{C}$ for $24 \mathrm{~h}$. The reaction was terminated at room temperature by the addition of distilled water and $\mathrm{H}_{2} \mathrm{O}_{2}(30 \%)$. The resultant solution was washed several times with $10 \%$ dilute $\mathrm{HCl}$ and distilled water in order to remove metal ions and centrifuged at $800 \mathrm{rpm}$ for $20 \mathrm{~min}$. The obtained graphite oxide powder was sonicated for $1 \mathrm{~h}$ to yield nanographene oxide. The resulting GO was freeze-dried to obtain as loose powder and stored for further use.

\section{CS functionalized GO}

Chitosan was attached on to the surface of GO via an amidation process [18] to form GO-CS. In brief, GO $(1 \mathrm{mg} / \mathrm{ml}, 50 \mathrm{ml})$ was first sonicated in distilled water $(50 \mathrm{ml})$ for $30 \mathrm{~min}$, EDC $(100 \mathrm{mg})$ and NHS $(75 \mathrm{mg})$ both are dissolved in water $(30 \mathrm{ml})$ were added to this GO dispersion under constant stirring in order to activate the carboxyl residues of GO and kept under constant stirring. To this mixture chitosan in acetic acid $(75 \mathrm{ml})$ was added dropwise and stirred at room temperature for $24 \mathrm{~h}$. The reacted product was centrifuged and washed several times with distilled water to remove the unreacted CS. The CS functionalized GO conjugate was freeze-dried and stored for further use.

\section{Synthesis of GO-CS-XG conjugate}

GO-CS-XG was synthesized by conjugating XG onto the surface of the intermediate product GO-CS. Initially, GO-CS $(100 \mathrm{mg})$ was dispersed in $100 \mathrm{ml}$ of distilled water and subjected to sonication followed by the addition of XG solution $(50 \mathrm{ml})$. The mixture was kept under magnetic stirring for $24 \mathrm{~h}$ at room temperature, then centrifuged and freeze-dried, resulting in the formation of GO-CS-XG.

\section{Loading of FU onto GO-CS-XG conjugate}

FU was loaded on to the GO-CS-XG system by simply mixing FU to GO-CS-XG solution followed by overnight stirring. The solution was centrifuged twice at $8000 \mathrm{rpm}$ for $20 \mathrm{~min}$ to remove the unbound $\mathrm{FU}$ retained in the supernatant. The final product GO-CS-XG-FU was lyophilized and stored for further use.

$$
\text { FU loading capacity (\%) } \frac{\text { Mass of loaded FU }}{\text { Mass of nanocarrier }} \times 100
$$

\section{Characterization techniques}

UV-Visible absorption spectra for the samples were performed using Perkin Elmer UV-Vis spectrophotometer (Model: Lambda 35). The samples were placed in a quartz cell. UV-Visible absorption spectra for these samples scanned in the wavelength ranging from 200 to $800 \mathrm{~nm}$. The FTIR analysis was done with a Perkin Elmer (spectrum two model) Fourier Transform Infrared spectrophotometer over the range of $400-4000 \mathrm{~cm}^{-1}$; powdered samples were mixed with potassium bromide and pelletized, which were used to confirm the presence of functional groups on the surface of GO, GO-CS, GO-CS-XG and GO-CS-XG-FU. Zeta potentials were measured using Horiba SZ 100 Nanopartica. All the Experiments were carried in triplicates. Morphological analysis of the samples were done by Scanning electron microscopy (Carl Zeiss Evo 18 model) and Transmission electron microscopy by (FEI Tecnai, G2 20Twin). TEM images were obtained by placing a drop of sample on a copper grid and evaporated at room temperature. Surface morphology and roughness of the samples were visualized by Atomic force microscopy (Nanosurf Easy Scan 2).

\section{Biocompatibility studies}

\section{In vitro hemolysis assay}

The hemolytic activity of GO-CS-XG, free fucoidan and fucoidanloaded nanocarrier was analyzed on Human red blood cells [19]. Fresh human RBCs were removed from $5 \mathrm{ml}$ of the citrated blood by centrifugation at $500 \mathrm{~g}$ for $10 \mathrm{~min}$. The cells were washed with phosphate-buffered saline (PBS) in order to remove the plasma and the process has been repeated 2 to 3 times to obtain serum-free blood cells and then it is resuspended in $20 \mathrm{ml}$ of PBS. A stock of $5 \%$ haematocrit suspension was used in this analysis. $100 \mu \mathrm{l}$ of diluted $\mathrm{RBC}$ suspension was treated with equal volumes of each sample at different concentrations $(20,40,60,80$ and $100 \mu \mathrm{g} / \mathrm{ml})$ and incubated for $30 \mathrm{~min}$ at $25^{\circ} \mathrm{C}$. After incubation, the mixture was centrifuged at $500 \mathrm{~g}$ for $10 \mathrm{~min}$ and the absorbance of the supernatant was determined at $540 \mathrm{~nm}$ using a microplate reader (Epoch Microplate; BioTek). RBCs suspended in 2\% Triton-X and PBS was used as the positive and negative controls respectively, the percentage of hemolysis of RBCs was calculated using the formula

$$
\text { Hemolysis }(\%)=100 \times \frac{(\text { OD sample }- \text { OD PBS })}{(\text { OD TritonX }- \text { OD PBS })}
$$

\section{Anti-inflammatory assay}

An anti-inflammatory activity was performed by the membrane stabilization method $[20,21]$. In brief, $5 \mathrm{ml}$ of whole blood was 
collected and mixed with equal volume of Alsever's solution and centrifugation at $500 \mathrm{~g}$ for $5 \mathrm{~min}$. The RBC pellets were further washed with isosaline and the same process was repeated for 3 times and then resuspended in water. Different concentrations $(20,40,60,80$ and $100 \mu \mathrm{g} / \mathrm{ml})$ of GO-CS-XG, FU, GO-CS-XG-FU were prepared to which $0.3 \mathrm{ml}$ of $\mathrm{HRBC}$ was added along with $0.3 \mathrm{ml}$ of PBS and $0.5 \mathrm{ml}$ of hypo saline, which was subjected to incubation at $37{ }^{\circ} \mathrm{C}$ for $30 \mathrm{~min}$. The suspension was then centrifuged at $3000 \mathrm{rpm}$ for $5 \mathrm{~min}$ and measured at $540 \mathrm{~nm}$ using a microplate reader (Epoch microplate spectrophotometer; BioTek).

\section{pH stimuli-responsive release study}

FU release profile was investigated under two different $\mathrm{pH}$ environments (PBS at pH 5.3 and pH7.4) at $37^{\circ} \mathrm{C}$ for a period of $50 \mathrm{~h}$. FU containing GO-CS-XG Nps ( $3 \mathrm{ml}$ ) was placed in a dialysis bag in 7 $\mathrm{ml}$ PBS and stirred continuously. At time intervals, $3 \mathrm{ml}$ of the release solution was taken out and fresh PBS was added into the medium to keep the volume constant. The release of Fu from GO-CSXG Nps was measured by UV-Vis spectroscopy at the wavelength of $262.6 \mathrm{~nm}$ using a calibration curve.

\section{Mechanism of FU release}

To investigate the drug release mechanism of loaded FU from GO-CSXG-FU, various models, including Zero order, First order, Higuchi, Korsmeyer-Peppas model, Hixson Crowell, were employed to fit the accumulative release data and to understand the drug release kinetics $[22,23]$.

\section{Zero-order model}

To observe the release kinetics, data obtained from in vitro FU release studies were plotted as cumulative percent of FU released $\left(\% \mathrm{Q}_{\mathrm{t}}\right)$ vs time $(\mathrm{t})$.

$$
Q_{t}=Q_{0}=K_{0} \mathrm{t}
$$

Where $Q_{0}$ is the initial amount of FU in solution, $Q_{t}$ is the amount of $\mathrm{FU}$ dissolved in time $\mathrm{t}$ and $\mathrm{K}_{0}$ is the zero-order release constant expressed in units of concentration/time.

\section{First-order model}

Release kinetics which followed the first order can be expressed by the equation:

$$
\log C=\log C_{0}-K t / 2.303
$$

Where $\mathrm{C}_{0}$ is the initial concentration of $\mathrm{FU}, \mathrm{K}$ is the first-order rate constant and $\mathrm{t}$ is the time. Hence the drug release kinetics data obtained from in vitro study are plotted as cumulative log percent of FU remaining vs time $(\mathrm{t})$.

\section{Higuchi model}

Higuchi equation can be represented in the simplified form

$$
\mathrm{Q}=\mathrm{K}_{\mathrm{H}} \times \mathrm{t}_{1 / 2}
$$

Where $\mathrm{K}_{\mathrm{H}}$ is the Higuchi dissolution constant. The data obtained were plotted against Cumulative percent drug released vs square root of time $(\sqrt{\mathrm{t}})$.

\section{Korsmeyer-peppas model}

60 percent of drug release data were fitted in the Korsmeyer-Peppas model and described by the following equation to observe the mechanism of drug release.

$$
\mathrm{M}_{\mathrm{t} / \mathrm{M}}=\mathrm{K}_{\mathrm{kp}} \mathrm{t}^{\mathrm{n}}
$$

Where $K_{k p}$ is the Korsmeyer release rate constant, $n$ is the release exponent, and $\mathrm{M}_{\mathrm{t}} / \mathrm{M}_{\infty}$ is the proportion of FU released at time $t$. The $\mathrm{n}$ value is used to differentiate between different releases. If $\mathrm{n}$ is 0.45 $\leq \mathrm{n}$, the diffusion mechanism is Fickian, $0.45<\mathrm{n}<0.89$ is non-Fickian, $\mathrm{n}=0.89$ is Case II transport, and $\mathrm{n}>0.89$ is super case II transport in this model [23]. A graph is displayed between cumulative log percent of FU released $\left(\log \mathrm{M}_{\mathrm{t}} \mathrm{M}_{\infty}\right)$ vs $\log$ time $(\log \mathrm{t})$ to evaluate release kinetics.
To study release kinetics, a graph is plotted between cumulative log percent of FU released (log) vs log time

\section{Hixson-crowell model}

Hixson-Crowell showed a relationship between drug release and time which can be expressed by the equation as follows

$$
\mathrm{W}_{0}{ }^{1 / 3}-\mathrm{W}_{\mathrm{t}^{1 / 3}}=\mathrm{K}_{\mathrm{HC}} \mathrm{t}
$$

Where $\mathrm{W}_{0}$ is the initial amount of FU in the pharmaceutical dosage form and $\mathrm{Wt}$ is the remaining amount of FU in the pharmaceutical dosage form at time $t$; $\mathrm{K}_{\mathrm{HC}}$ is the Hixson-Crowell constant relating to the surface volume. To investigate the release kinetics, data from in vitro drug release were plotted against time as the cube root of drug $\%$ remaining in matrix.

\section{Cytotoxicity study}

The in vitro cytotoxicity of prepared samples was studied on human histiocytic lymphoma cells, U 937 by MTT assay.

\section{MTT cytotoxicity assay}

A cell count of $10^{5} \mathrm{U} 937$ cells was seeded in 96-plates for 24h. After $24 \mathrm{~h}$ of cell adhesion, the cells were treated with different concentrations $(20-100 \mu \mathrm{g} / \mathrm{ml})$ of GO-CS-XG, free FU, GO-CS-XG-FU to evaluate the cytotoxicity of the prepared nanocarrier and FU loaded nanocarrier and further incubated for 24,48 and $72 \mathrm{~h}$ respectively. After incubation, the medium was replaced with $100 \mu \mathrm{l}$ of MTT in PBS and subjected to further incubation of $4 \mathrm{~h}$ at $37{ }^{\circ} \mathrm{C}$. Finally, the medium was removed and the formazan crystals formed in the cells were dissolved in $100 \mu$ l of DMSO. In a microplate reader, light absorbance was measured at $570 \mathrm{~nm}$. Cell viability was calculated as a percentage relative to untreated control.

\section{Statistical analysis}

Statistical analysis was performed using Graph pad prism 9 software. All the measurements were repeated thrice and data were expressed as mean \pm SD. Data were analyzed using a one-way analysis of variance (ANOVA). A " $p$ " value of less than $0.05(p<0.05)$ was considered to be statistically significant.

\section{RESULTS AND DISCUSSION}

\section{UV-Visible analysis}

The UV-Vis spectra of GO, GO-CS, GO-CS-XG and GO-CS-XG-FU are shown in fig. 1a-d respectively. The UV visible spectrum thus obtained gives a characteristic peak at $230 \mathrm{~nm}$ for GO nanoparticles ascribed to a $\pi-\pi^{*}$ transitions of-C=C-bonds [24], with a slight rise in peak at $265 \mathrm{~nm}$ which corresponds to the $n-\pi^{*}$ transitions of $-\mathrm{C}=0$-bond (fig. 1a), and these peaks slightly shifted to a single peak at $260 \mathrm{~nm}$ (fig. 1b) when the surface of the GO was functionalized with chitosan. Binding of xyloglucan on to the surface of GO-CS (fig. 1c) was observed by a peak at $281 \mathrm{~nm}$. It was observed that the loading of FU exhibited a strong peak at $275 \mathrm{~nm}$, indicating that the FU drug was successfully conjugated on the nanocarrier system GO-CS-XG nanoparticles (fig. 1d).

\section{FTIR spectroscopy}

The functional groups and interactions of GO, GO-CS, GO-CS-XG and GO-CS-XG-FU were confirmed by FT-IR analysis (fig. 2 and 3). FT-IR spectrum of GO (fig. 2) showed the absorption peak at $1740 \mathrm{~cm}^{-1}$, $1636 \mathrm{~cm}^{-1}$ and $1400 \mathrm{~cm}^{-1}$ are attributed to the $\mathrm{C}=0$ stretching vibration, $\mathrm{C}=\mathrm{C}$ stretching vibration of the $\mathrm{sp}^{2}$ carbon skeletal or epoxy groups and $\mathrm{O}-\mathrm{H}$ deformation vibrations of ketone groups, respectively. The peak at $3431 \mathrm{~cm}^{-1}$ and $2918 \mathrm{~cm}^{-1}$ clearly states $\mathrm{O}-\mathrm{H}$ stretching vibration and the saturated $\mathrm{C}-\mathrm{H}$ bond vibration. The weak band at $1200 \mathrm{~cm}^{-1}$ and two sharp peaks at $1103 \mathrm{~cm}^{-1}$ and $1029 \mathrm{~cm}^{-1}$ were due to the $\mathrm{C}-\mathrm{OH}$ stretching and the vibrations of epoxy (C-O-C) groups present at the edges of the GO planes. These results of GO showed that ample of hydroxyl, carboxyl and epoxy groups were presented on the surface of GO [25].

In the FT-IR spectrum of GO-CS (fig. 2), the shifts in the characteristic absorption peaks revealed that these alterations could be related to the reaction of GO carboxyl groups with the amino groups of chitosan [26] in order to form an amide I at $1623 \mathrm{~cm}^{-1}$, in addition to this, a 
band at $1567 \mathrm{~cm}^{-1}$ (amide II) is a characteristic of the N-H bonds of Nacetyl groups. Presence of carboxylate groups at $1400 \mathrm{~cm}^{-1}$. The prominent peak at $1740 \mathrm{~cm}^{-1}$, indicating the presence of- $\mathrm{COOH}$ group at $1740 \mathrm{~cm}^{-1}$ in GO is almost shifted to $1720 \mathrm{~cm}^{-1}$. These observations clearly indicate the functionalization of GO with CS.

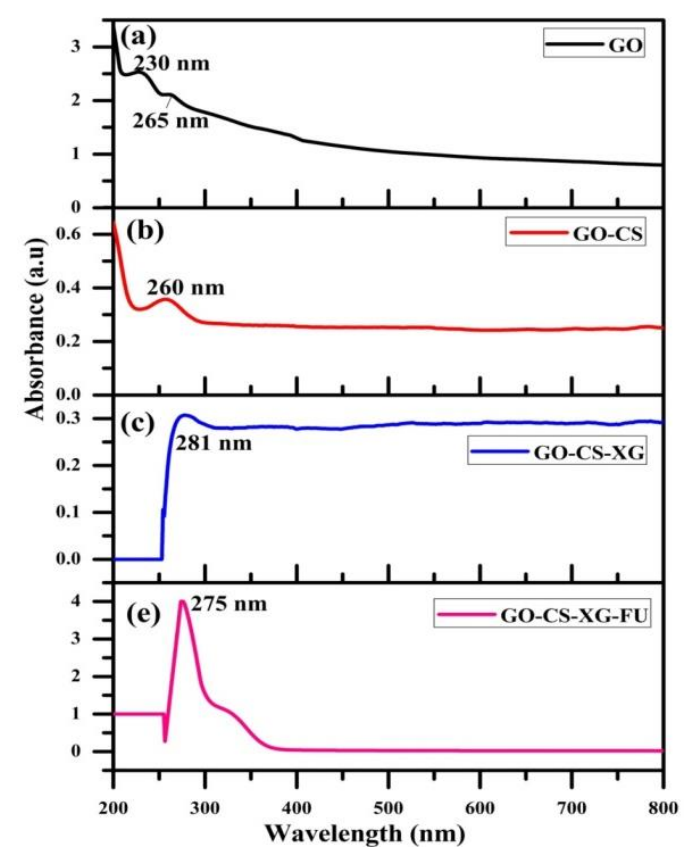

Fig. 1: UV-Visible absorption spectra of (a) GO, (b) GO-CS, (c) GO-CS-XG, (d) GO-CS-XG-FU

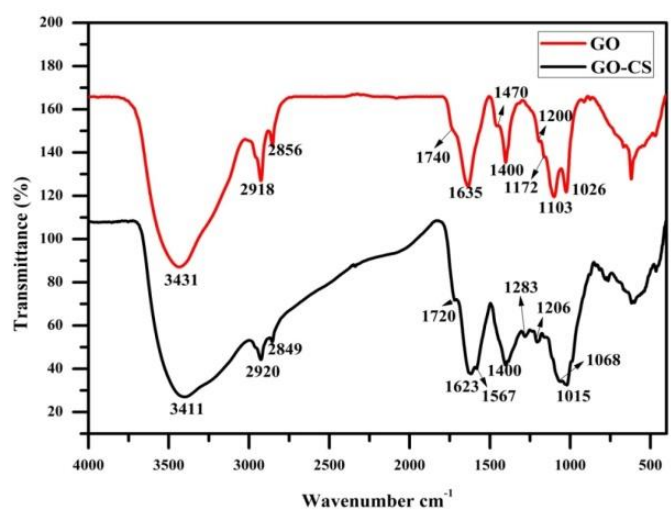

Fig. 2: FT-IR spectra of (a) GO, (b) GO-CS
The FT-IR spectra of GO-CS-XG and GO-CS-XG-FU are shown in fig. 3. Conjugation of xyloglucan to GO-CS (fig. 3) results in the ring opening, which leads to the formation of amide linkage between the aldehyde group of xyloglucan units and free amino groups present on the surface of GO-CS and the peak at $1720 \mathrm{~cm}^{-1}$ for-CO groups disappeared. A broad absorption band at $3431 \mathrm{~cm}^{-1}$ attributed to the hydroxyl $(0-\mathrm{H})$ stretching. A band at $2921 \mathrm{~cm}^{-1}$ to $2843 \mathrm{~cm}^{-1}$ is due to the asymmetric vibration of the carbohydrate ring- $\mathrm{C}-\mathrm{H}$ bonds. The region from 1239 to $1030 \mathrm{~cm}^{-1}$ is typical of xylose $[26,27]$, corresponding to the $\mathrm{C}-\mathrm{OH}$ vibrations of the glycosidic $\mathrm{C}-\mathrm{O}-\mathrm{C}$ bonds. It is clearly evident that the xyloglucan was effectively conjugated to the GO-CS system via intermolecular or ionic interactions.

Furthermore, the loading of FU on to the GO-CS-XG nanocarrier system (fig. 3) shows the presence of peaks at $1243 \mathrm{~cm}^{-1}$ and $850 \mathrm{~cm}$ 1 indicates the stretching vibrations of $-\mathrm{SO}_{3}$ groups and the bending vibration of C-O-S, thus depicting the successful loading of drug with the GO-CS-XG nanocarrier system by means of non-covalent binding to form GO-CS-XG-FU.

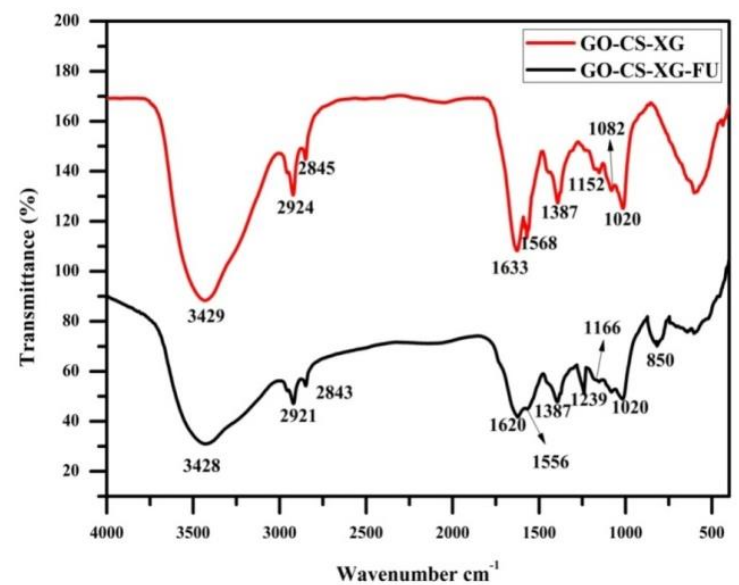

Fig. 3: FT-IR spectra of (c) GO-CS-XG, (d) GO-CS-XG-FU

\section{Surface morphological studies}

\section{Scanning electron microscopy (SEM)}

SEM images of GO, GO-CS, GO-CS-XG and GO-CS-XG-FU (fig 4a-d). GO exhibits typical stacked flakes closely associated with each other. Functionalization of GO with CS showed a stacked and wrinkled flakes-like structure, whereas the SEM images of GO-CS-XG were porous in nature and GO-CS-XG-FU clearly depicts the embedding of $\mathrm{FU}$ onto the porous surface of GO-CS-XG leads to the agglomeration of nanoparticles.
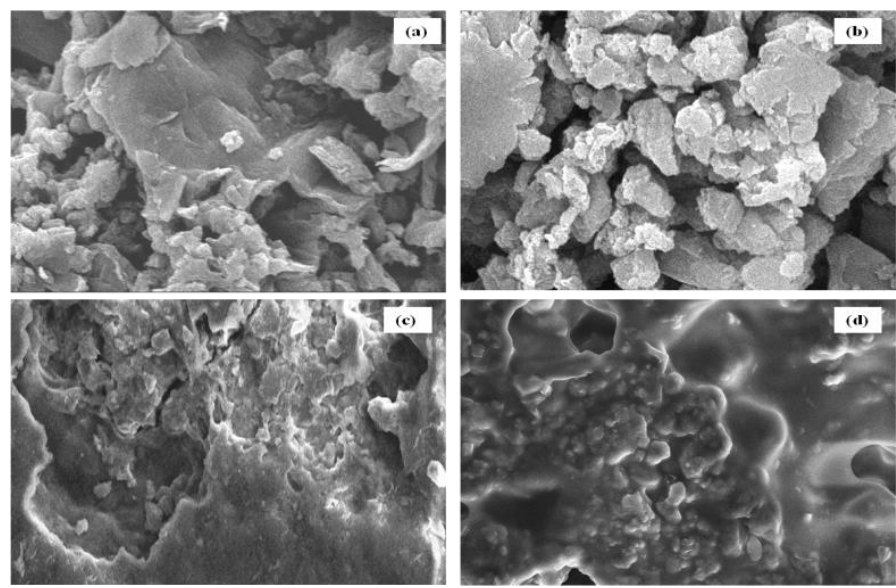

Fig. 4: SEM images of (a) GO, (b) GO-CS, (c) GO-CS-XG, (d) GO-CS-XG-FU 


\section{Transmission electron microscopy (TEM)}

TEM images of GO, GO-CS, GO-CS-XG and GO-CS-XG-FU (fig. 5a-d). GO nanoparticles (fig. 5a) showed a crumpled flake with its intrinsic folding. GO-CS showed some morphologies different from the GO, a layered sheath-like structure indicating a proper adhesion between GO and chitosan matrix (fig. 5b). TEM image of GO-CS-XG and GO-CS-XG-FU (fig. $5 \mathrm{c}$ and $5 \mathrm{~d}$ ) were found to be wrinkled with particles embedded within the surface. From the TEM analysis it is clear that this nanocarrier system GO-CS-XG brings an intrinsic change in its structure upon functionalization, conjugation and loading of drug.

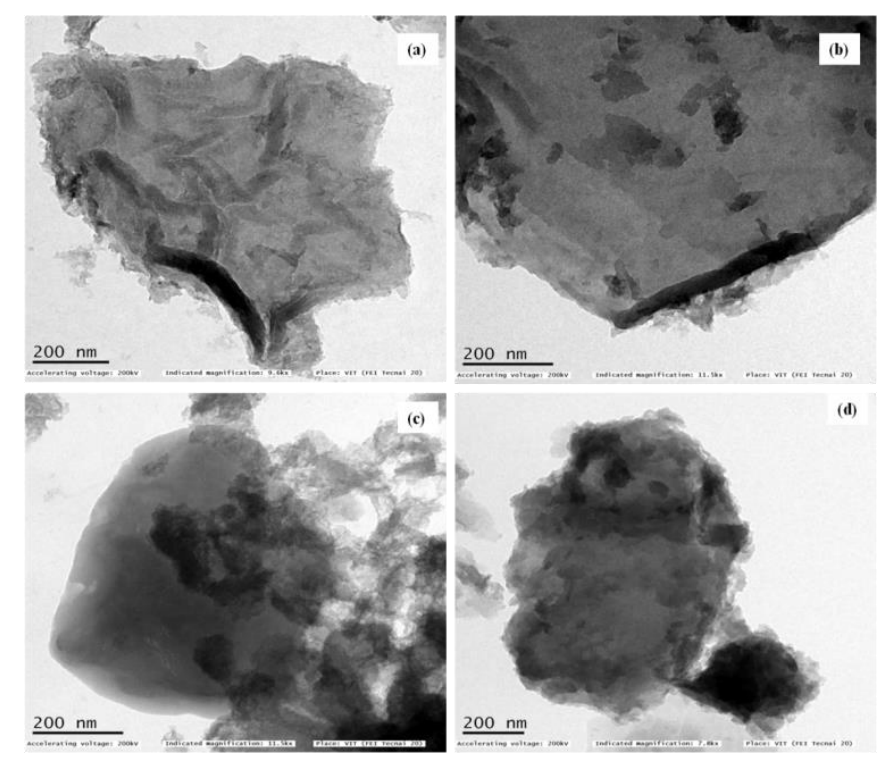

Fig. 5: TEM images of (a) GO, (b) GO-CS, (c) GO-CS-XG, (d) GO-CS-XG-FU

\section{Atomic force microscopy (AFM)}

AFM images of the nanocarrier system GO, GO-CS and GO-CS-XG-FU are shown in fig. 6a-c. It gives an idea about the surface morphology of the prepared samples. It is observed that the average thickness of the GO is 0 to $5 \mu \mathrm{m}$. Fig. $6 \mathrm{a}$ depicts the three-dimensional image of GO and the surface is layer by layer like morphological (wave-like morphology) of GO. The corresponding two-dimensional structure were clearly indicates the layer by layer morphological structure. The stacking of GO layers together during sample preparation for AFM investigation causes the variance in GO thickness. Fig. $6 \mathrm{~b}$ and 6c exhibits the AFM images of GO-CS and GO-CS-XG-FU. GO-CS showed a mean surface roughness of about $125 \mathrm{~nm}$ for $4.98 \mu \mathrm{m} \mathrm{x}$ $4.98 \mu \mathrm{m}$ and $48.2 \mathrm{~nm}$ for GO-CS-XG-FU at $10.1 \mu \mathrm{m} \times 10.1 \mu \mathrm{m}$. After the functionalization, the surface morphology of GO-CS changed, which was indicated in accordance with the TEM images.

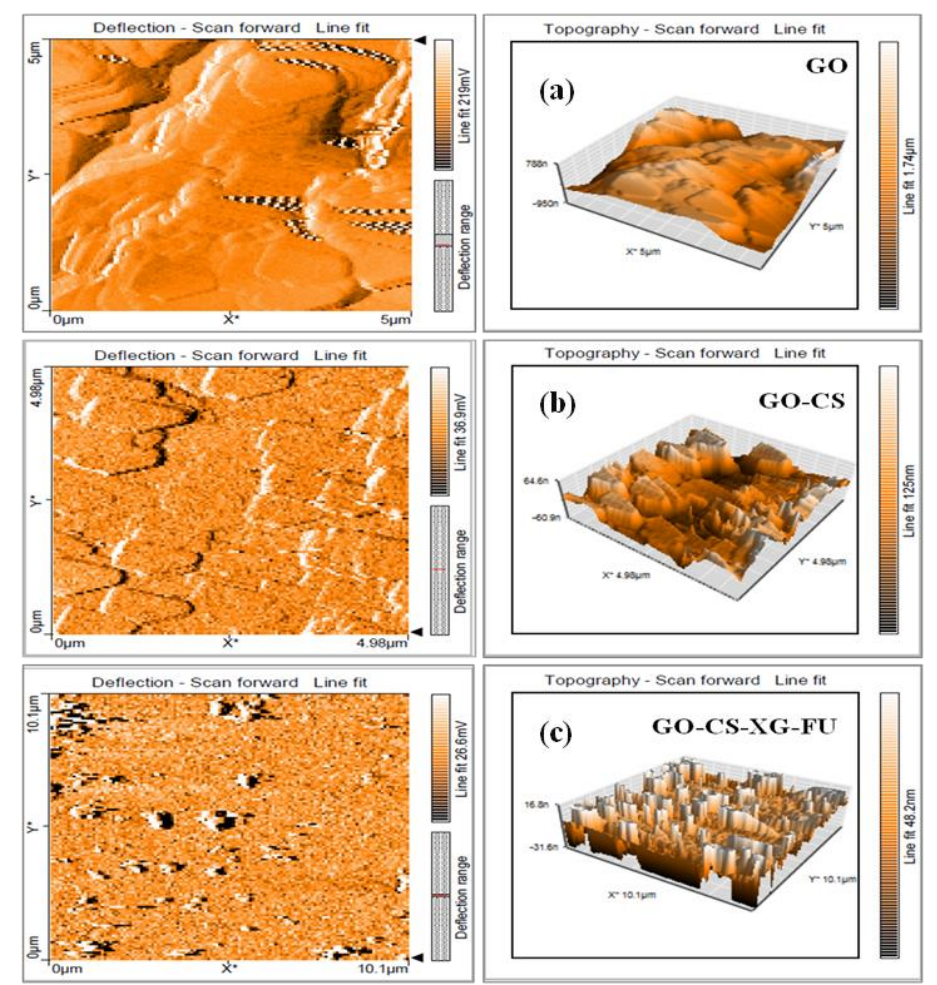

Fig. 6: AFM images of (a) GO, (b) GO-CS, (c) GO-CS-XG-FU 


\section{Drug loading and encapsulation efficiency}

FU was loaded onto the GO-CS-XG nanocarrier via a simple mixing and sonication method. The unbound drug was removed by centrifugation and the loading capacity and entrapment efficiency of FU on GO-CS-XG nanocarrier were calculated by measuring the concentration of unbound drug-using UV-Vis at $262.6 \mathrm{~nm}$ included in table 1. Based on the results the entrapment efficiency and loading capacity of GO-CS-XG-FU are higher than the others and are as high as $75.79 \%$ and $83.45 \%$, respectively, which may be due to the ionic interactions between FU and the GO-CS-XG. As a result, GO-CS-XG appears to be a promising drug carrier candidate.

\section{Table 1: Results of loading and efficiency of FU on GO-CS and} GO-CS-XG

\begin{tabular}{llc}
\hline Material & Drug loading* & Entrapment efficiency* \\
\hline GO-CS & $48 \pm 0.8$ & $44.5 \pm 0.6$ \\
GO-CS-XG & $75.7 \pm 0.56$ & $83.4 \pm 1.48$ \\
\hline
\end{tabular}

*Data represented in table are mean \pm SD for three replicate experiments $(n=3)$.

\section{Zeta potential}

Further evidence for the successful functionalization and loading of FU to the nanocarrier GO-CS-XG was analyzed in the zeta potential of the sample; fig. 7a-d shows the zeta potential values obtained for the GO-CS-
XG-FU nanosystem at varies stages of synthesis. The Zeta potential of GOCS (average value $26.8 \pm 0.82 \mathrm{mv}$ ) became positive after conjugation with GO (average value- $24.4 \pm 0.7 \mathrm{mv}$ ). The cationic polysaccharide CS shielded the negative charge of the graphene oxide nanoparticles and offsets its charge. After functionalization of GO-CSNps with the neutral charge polysaccharide XG, surface zeta potential changed to an average value of$20.4 \pm 0.37 \mathrm{mv}$ indicating the conjugation of XG with the GO-CSNps. Changes in the zeta potential of GO-CS-XG-FU with an average value of$15.9 \pm 0.9 \mathrm{mv}$ due to the conjugation of sulphated polysaccharide fucoidan, thus indicating successful loading of the FU drug onto the surface of GOCS-XG nanoparticles.

\section{In vitro hemolysis assay}

In this study, the hemocompatibilty was evaluated using human blood in terms of hemolysis. The in vitro hemolytic assay was evaluated for each sample (FU, GO-CS-XG, GO-CS-XG-FU) at five different concentrations; 20,40,60,80 and $100 \mu \mathrm{g} / \mathrm{ml}$. Triton X-100 and PBS were used as positive and negative controls, respectively. Fig. 8 shows the incubation of RBCs with GO-CS-XG, free FU and GOCS-XG-FU for $0.5 \mathrm{~h}$. In case of FU the \% hemolytic activity was recorded as 25.34 at a concentration of $100 \mu \mathrm{g} / \mathrm{ml}$, whereas in the case of GO-CS-XG and GO-CS-XG-FU, the hemolytic activity was recorded at $10.50 \%$ and $3.75 \%$ at a concentration of $100 \mu \mathrm{g} / \mathrm{ml}$, thus indicating that the drug-loaded nanocarrier system considered to be non-hemolytic which suggest that GO-CS-XG-FU nanoparticles possess good blood compatibility. Fluorescence microscopic observations of RBCs treated with PBS, GO-CS-XG, FU and GO-CS-XGFU are depicted in the fig. 9 in accordance with the hemolytic assay.
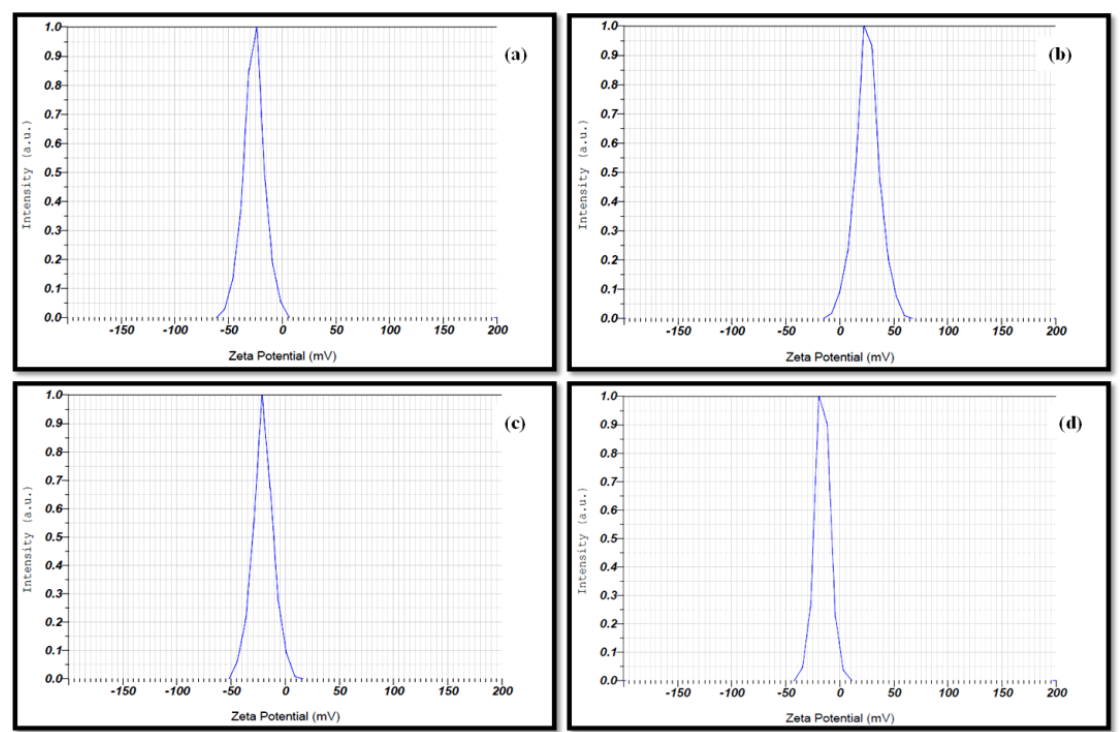

Fig. 7: Zeta potential of (a) GO, (b) GO-CS, (c) GO-CS-XG, (d) GO-CS-XG-FU. Data represented in fig. are mean \pm SD for three replicate experiments $(n=3)$

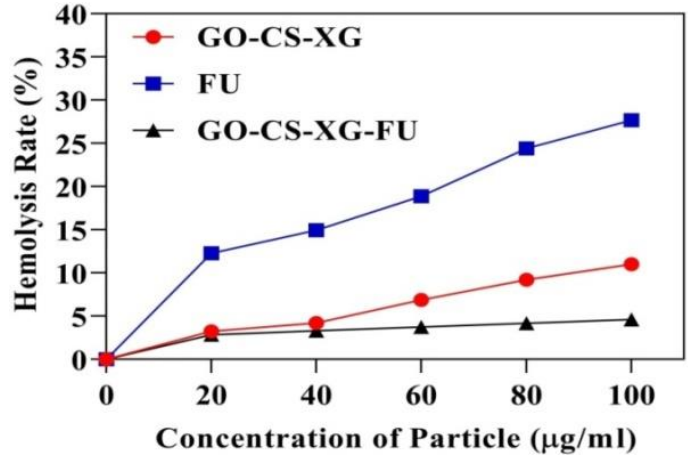

Fig. 8: Percentage hemolysis of GO-CS-XG, FU and GO-CS-XG-FU. Data represent mean $\pm \operatorname{SD}(n=3)$

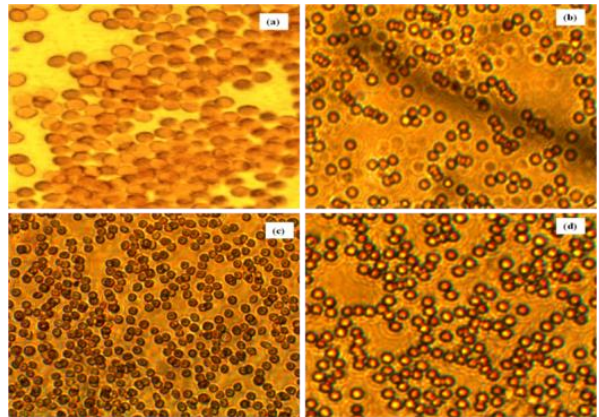

Fig. 9: Fluorescence microscopy images of erythrocyte structure: (a) RBC in PBS, (b) RBC incubated with GO-CS-XG, (c) RBC incubated with FU, (d) RBC incubated with GO-CS-XG-FU 


\section{Anti-inflammatory assay}

The predominant basis for a nanoparticle to be biocompatible is it shouldn't cause any inflammation. Anti-inflammatory activity was evaluated by human red blood cells membrane stabilization method. GO-CS-XG, FU and GO-CS-XG-FU showed $82.56 \%, 86.23 \%$ and $90.62 \%$ stabilization at a concentration of $100 \mu \mathrm{g} / \mathrm{ml}$, respectively in accordance with diclofenac sodium salt as standard (fig. 10). Thus percentage of stabilization increases with increase in concentration.

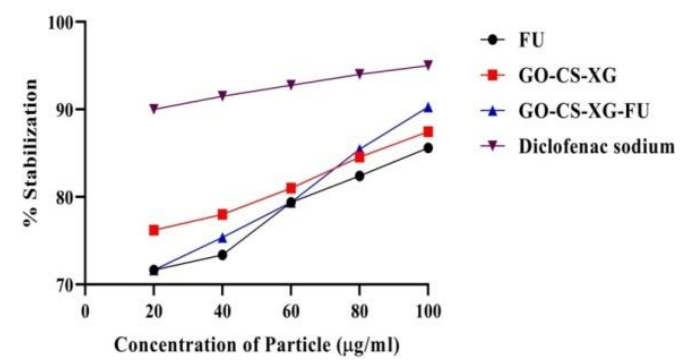

Fig. 10: Anti-inflammatory activity of FU, GO-CS-XG, GO-CS-XGFU and diclofenac sodium (Standard). Each value represents the mean $\pm \operatorname{SD}(n=3)$

\section{pH stimuli-responsive release studies}

In order to enhance the release of anti-cancer drug within the tumor cells and to avoid the undesired release in blood circulation, it is prominent to have a $\mathrm{pH}$-responsive drug release from the nanocarrier system. Therefore it is important to investigate controlled drug release from the fucoidan-loaded GO-CS-XG based nanocarriers at $37{ }^{\circ} \mathrm{C}$ in different $\mathrm{pH}$ environments (PBS at $\mathrm{pH} 5.3$ and $\mathrm{pH}$ 7.4) for a period of $60 \mathrm{~h}$ (fig. 11).

In the current study, we have chosen fucoidan (FU) as a model drug extracted from the marine brown algae Sargassum wightii to exhibit the release from a xyloglucan mediated GO-CS nanocarrier. As seen in fig. 11 at physiological $\mathrm{pH}$, there was a slower release rate at controlled manner in FU from GO-CS-XG nanocarrier was only $30 \%$ of the total bound $\mathrm{FU}$ after $60 \mathrm{~h}$, which can due to the ionic interactions between the drug $\mathrm{FU}$ and the GO-CS-XG nanocarrier system. In case of acidic condition ( $\mathrm{pH} 5.3$ ), the release rate of $\mathrm{FU}$ was dramatically enhanced to $70 \%$, leading to a higher preferred release of FU, it means that the drug-loaded wasn't released fully even after $60 \mathrm{hr}$ still $30 \%$ of the drug is retained in the system for circulation. Thus GO-CS-XG nanocarrier system has a significant improvement in prolonged releasing which can sustain up to $60 \mathrm{~h}$.

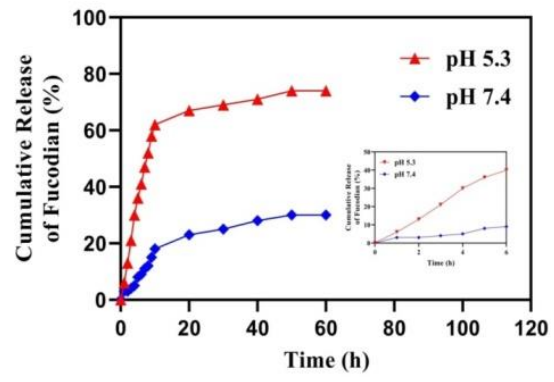

Fig. 11: $\mathrm{pH}$-responsive in vitro release profile of FU from GO-CSXG-FU drug carrier at $37^{\circ} \mathrm{C}$ in PBS (pH 5.3 and pH 7.4) by UVVis analysis. Each value represents the mean $\pm \operatorname{SD}(n=3)$
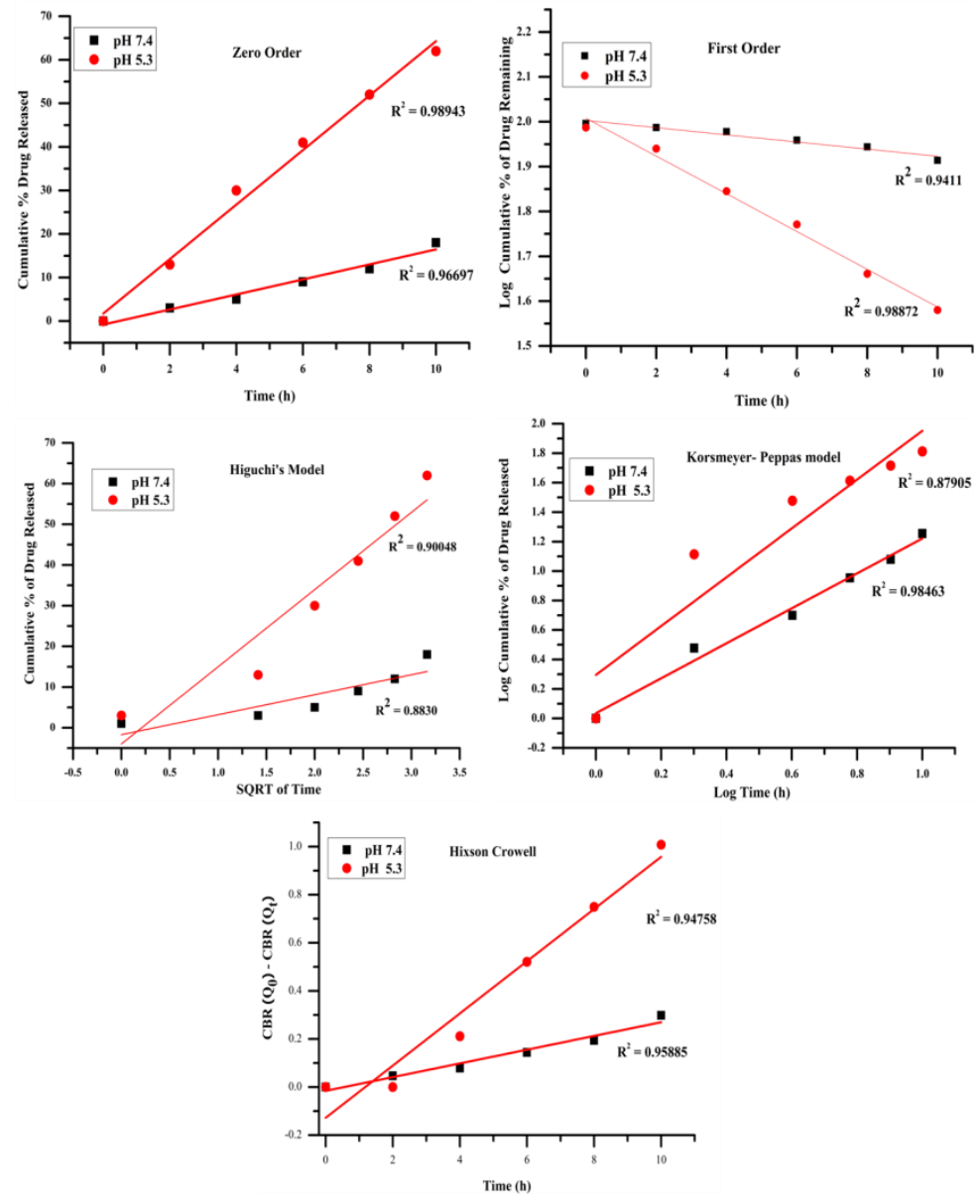

Fig. 12: The kinetic equation models of the zero-order, first-order, higuchi, korsmeyer-peppas model and hixson crowell of drug release from the FU-loaded GO-CS-XG system 


\section{Fu release kinetics mechanism}

To investigate the kinetics and release mechanism of FU from GOCS-XG-FU formulation, various kinetic models were applied-Zero order, First order, Higuchi, Hixson Crowell and Korsmeyer-Peppas model at $\mathrm{pH} 7.4$ and $\mathrm{pH} 5.3$ respectively and interpreted in the form of graphical presentation and evaluated by correlation coefficient $\left(\mathrm{R}^{2}\right)$ represented in fig. 12a-e. The obtained release parameters are also listed in table 2 . The best-fitted model was determined based on $\mathrm{R}^{2}$ value. The kinetic models that follow the drug release are determined by the highest degree of correlation coefficient $[22,23]$. From the results, it was found that zero order kinetics $(0.9669$ and
0.9894 for $\mathrm{pH} 7.4$ and $\mathrm{pH}$ 5.3) and Hixson-Crowell model (0.9588 and 0.9475 for $\mathrm{pH} 7.4$ and $\mathrm{pH}$ 5.3) showed a higher degree of correlation coefficient compared to other models. Hence drug release profile of FU follows a constant drug release and the level of drug in the blood remains constant throughout the delivery.

In addition, the $\mathrm{n}$ value of Korsmeyer-Peppas model can be used to characterize the release mechanism of FU. In this model, the release mechanism of $\mathrm{FU}$ at $\mathrm{pH} 7.4$ (R2 = 0.98463) implies that the drug release from the system follows anomalous (non-fickian) diffusion $(\mathrm{n}=0.643)$ and at $\mathrm{pH} 5.3(\mathrm{R} 2=0.87905)$ follows the fickian diffusion mechanism $(\mathrm{n}=0.41)$.

Table 2: Release parameters of FU from GO-CS-XG-FU obtained from 5 types of kinetic models at pH 7.4 and pH 5.3

\begin{tabular}{|c|c|c|c|c|c|c|}
\hline Sample code & pH & Zero-order & First-order & Higuchi model & Hixson Crowell & Korsmeyer-peppas model \\
\hline \multirow[t]{3}{*}{ GO-CS-XG-FU } & & $\mathrm{R}^{2}$ & & & & $\mathrm{n} \quad \mathrm{R}^{2}$ \\
\hline & 5.3 & 0.98943 & 0.9887 & 0.90048 & 0.94754 & 0.87905 \\
\hline & 7.4 & 0.96697 & 0.9411 & 0.88302 & 0.95585 & 0.98463 \\
\hline
\end{tabular}

\section{In vitro cytotoxicity}

The cytotoxicity of FU-loaded GO-CS-XG based pH-responsive nanocarrier system, free FU and GO-CS-XG were evaluated by MTT assay against human histiocytic lymphoma (U 937) cells for periods of $24 \mathrm{~h}, 48 \mathrm{~h}$ and $72 \mathrm{~h}$.

As depicted in fig. $13 \mathrm{a}-\mathrm{c}$, the cell viability decreased as the incubation time increased, thus exhibiting time-dependent cytotoxicity. Upon $24 \mathrm{~h}$ of exposure, the GO-CS-XG-FU showed $33 \%$ of cell death when compared to free FU, which shows a $20 \%$ cell death at a concentration of $100 \mu \mathrm{g} / \mathrm{ml}$, almost $13 \%$ of the efficacy of FU is increase when FU loaded on to GO-CS-XG nanocarrier system. After $72 \mathrm{~h}$ of incubation, the cell viability of GO-CS-XG-FU was around $18.3 \%$, almost $82 \%$ of cells died at a concentration of $100 \mu \mathrm{g} / \mathrm{ml}$. From the cytotoxicity results, it can be understood that drug-loaded GO-CS-XG-FU showed significant cytotoxicity against U 937 cells compared to FU and GO-CSXG. Hence the development of the GO-CS-XG nanocarrier system has the potential for targeted delivery of cancer drugs, thus proving the efficacy of FU as a suitable candidate through this nanocarrier system. Morphological changes in U 937 cells are shown in fig. 14a-b.
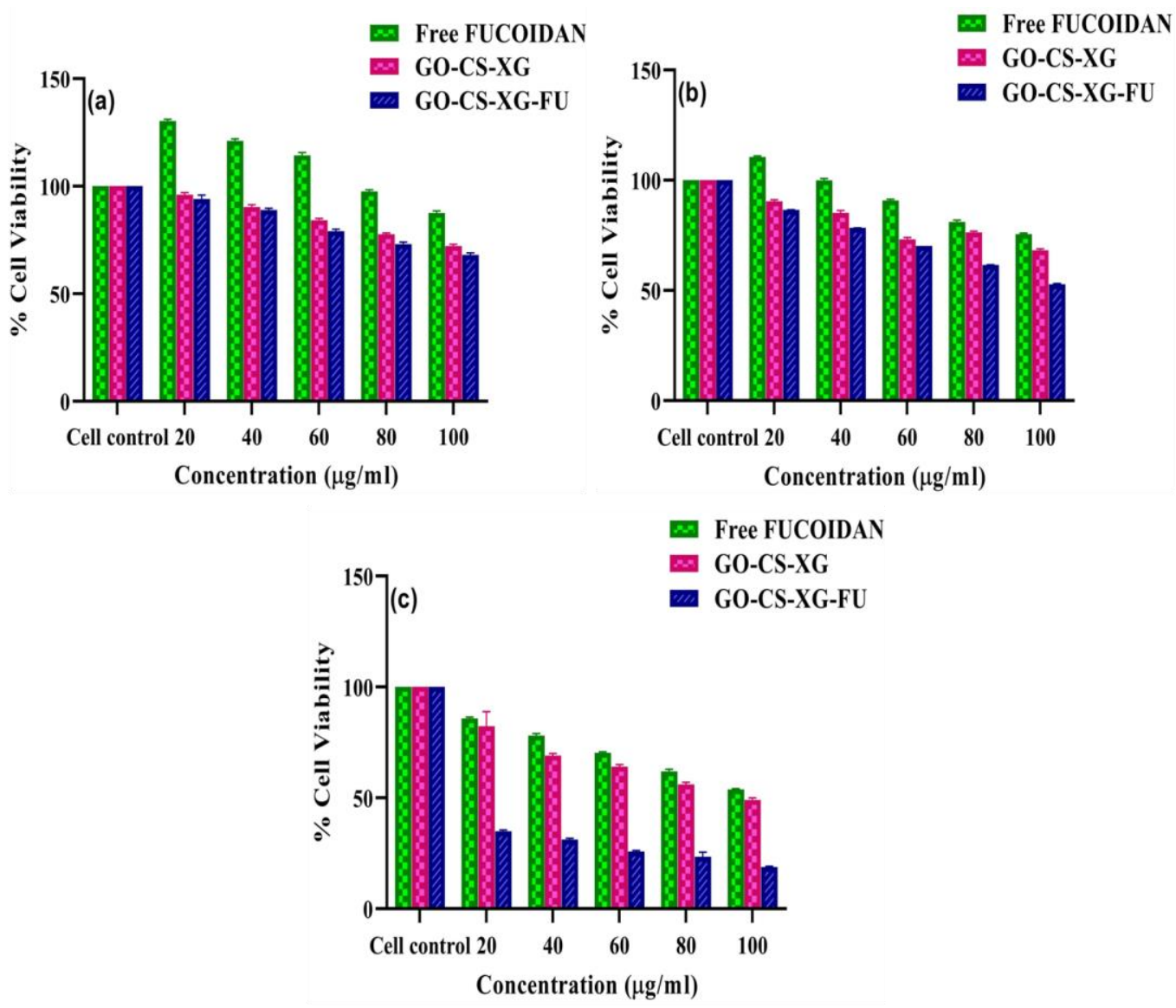

Fig. 13: Cytotoxicity of $U 937$ cells determined by MTT assay after $24 \mathrm{~h}(\mathrm{a}), 48 \mathrm{~h}$ (b) and $72 \mathrm{~h}$ (c) of exposure to different concentra tions of free fucoidan, GO-CS-XG and GO-CS-XG-FU. ( ${ }^{*}$ Data represent mean \pm SD $(n=3)$ for three replicate experiments; Significance at $5 \%$ level $(\mathbf{P}<\mathbf{0 . 0 5 )}$ 
(a) CONTROL

FU

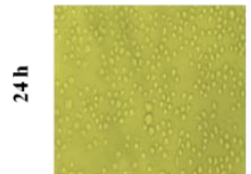

$\stackrel{5}{+}$

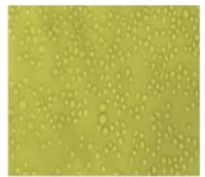

$\stackrel{1}{N}$

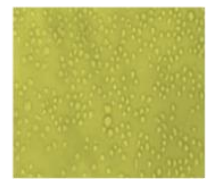

CONTROL

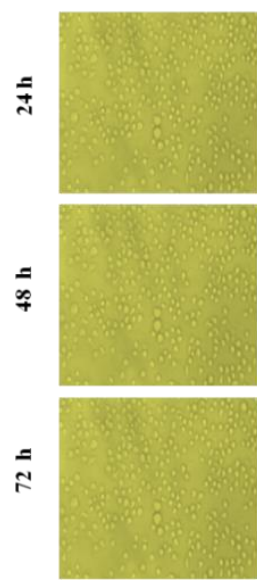

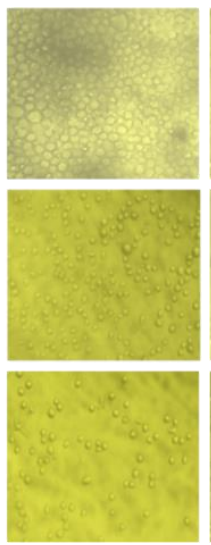

FU

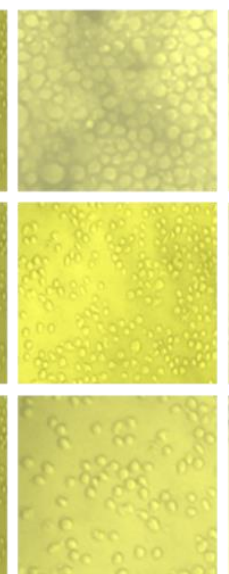

GO-CS-XG

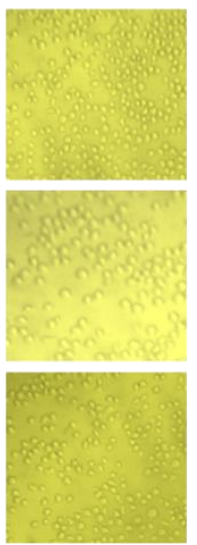

GO-CS-XG

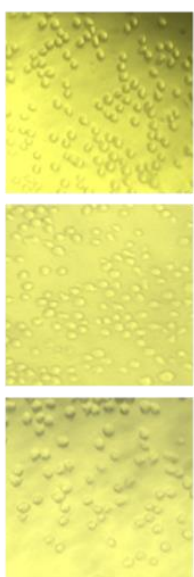

GO-CS-XG-FU

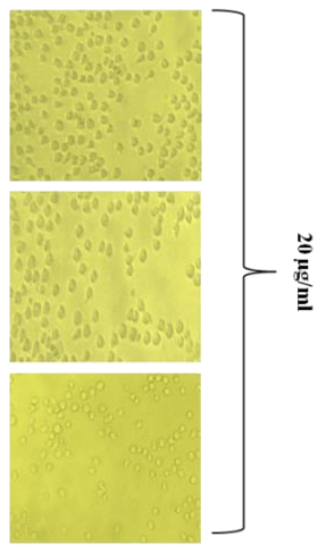

GO-CS-XG-FU

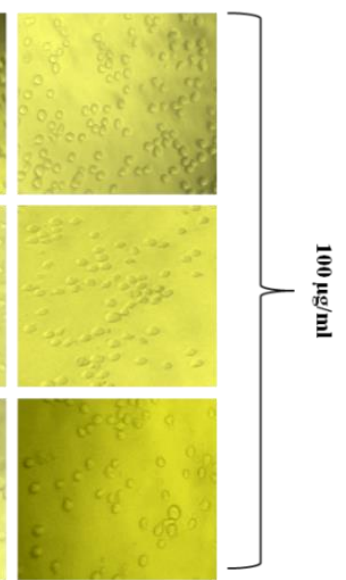

Fig. 14: Photomicrographs of $\mathrm{U} 937$ cells-(a) Cells treated with control, FU, GO-CS-XG and GO-CS-XG-FU at 20 $\mu$ g/ml and (b) Cells treated with control, FU, GO-CS-XG and GO-CS-XG-FU at $100 \mu \mathrm{g} / \mathrm{ml}$ upon $72 \mathrm{~h}$ of exposure

\section{CONCLUSION}

In conclusion, we have developed a promising platform for anticancer drug delivery system with high dug loading and efficiency. To specifically target the cancer cells, chitosan functionalized graphene oxide was conjugated with the targeting ligand xyloglucan The targeted and $\mathrm{pH}$-responsive GO-CS-XG drug delivery was loaded with fucoidan, an anticancer model drug extracted from marine brown algae Sargassum wightii. The in vitro release of FU was at a faster and higher rate in the case of acidic medium compared to physiological conditions. This $\mathrm{pH}$-sensitive drug release with high entrapment efficiency makes this nanocarrier system a prominent candidate for targeted drug delivery system. Hemolysis studies provide excellent biocompatibility by showing no toxic effect on RBCs. Better stabilization was achieved via an antiinflammatory assay. FU-loaded GO-CS-XG drug delivery systems were tested for cytotoxicity activity against human lymphoma (U937) cells exhibited higher cytotoxicity of $82 \%$ at higher concentration upon exposure to a period of $72 \mathrm{~h}$, which is well defined from the MTT assay. Therefore this present study is brief screening of polysaccharides as a new drug carrier system along with GO for targeted drug delivery.

\section{ACKNOWLEDGEMENT}

This research has no acknowledgment.

\section{FUNDING}

This research received no external funding.

\section{AUTHORS CONTRIBUTIONS}

All the authors have contributed equally.

\section{CONFLICT OF INTERESTS}

The authors confirm that this article has no conflict of interest

\section{REFERENCES}

1. Xie X, Luo S, Mukerabigwi JF, Mei J, Zhang Y, Wang S, et al. Targeted nanoparticles from xyloglucan-doxorubicin conjugate loaded with doxorubicin. RSC Adv 2016;6:26137-46.

2. Luyts K, Napierska D, Nemery B, Hoet PHM. How Physicochemical characteristics of nanoparticles cause their toxicity: complex and unresolved interrelations. Environ Sci Proc Impact 2013; 15:23-38.

3. Mazzarino L, Loch Neckel G, Dos Santos Bubniak L, Ourique F, Otsuka I, Halila S, et al. Nanoparticles made from xyloglucanblock-polycaprolactone copolymers: safety assessment for drug delivery. Toxicol Sci 2015;147:104-15.

4. Borandeh S, Abdolmaleki A, Abolmaali SS, Tamaddon AM. Synthesis, structural and in vitro characterization of $\beta$ cyclodextrin grafted L-phenylalanine functionalized graphene oxide nanocomposite: a versatile nanocarrier for $\mathrm{pH}$-sensitive doxorubicin delivery. Carbohydr Polym 2018;201:151-61.

5. Liu J, Cui L, Losic D. Graphene and graphene oxide as a new nanocarrier for drug delivery applications. Acta Biomater 2013;9:9243-57.

6. Pawde DM, Viswanadh MK, Mehata AK, Sonkar R, Narendra, Poddar S, et al. Mannose receptor-targeted bioadhesive chitosan nanoparticles of clofazimine for effective therapy of tuberculosis. Saudi Pharm J 2020;28:1616-25.

7. Da Silva ACC, De Almeida RR, Da Cruz Sousa AC, Martinez FNA, Denardin JC, Morais SM, et al. Xyloglucan-based hybrid nanocomposite with potential for biomedical applications. Int J Biol Macromol 2021;168:722-32. 
8. Mishra A, Malhotra AV. Graft copolymers of xyloglucan and methyl methacrylate. Carbohydr Polym 2012;87:1899-904.

9. Kulkarni AD, Joshi AA, Patil CL, Amale PD, Patel HM, Surana SJ, et al. Xyloglucan: a functional biomacromolecule for drug delivery applications. Int J Biol Macromol 2017;104:799-812.

10. Manivasagan $\mathrm{P}, \mathrm{Oh} \mathrm{J}$. Marine polysaccharide-based nanomaterials as a novel source of nanobiotechnological applications. Int J Biol Macromol 2016;82:315-27.

11. Palanisamy S, Vinosha M, Marudhupandi T, Rajasekar P, Prabhu NM. In vitro antioxidant and antibacterial activity of sulfated polysaccharides isolated from Spatoglossum asperum. Carbohydr Polym 2017;170:296-304.

12. Chidambararajan P, Keerthana V, Priyadharshini K, Sakthivel B. In vitro antioxidant and anticancer activity of Ulva lactuca $L$. using MOLT-3 cell line. Asian J Pharm Clin Res 2019;12:75-8

13. Sreekala Kannikulathel Gopidas, Nagaraj Subramani. In vitro antioxidant and cytotoxic properties of fucoidan from three indian brown seaweeds. Asian J Pharm Clin Res 2019;12:99-105.

14. Wang $\mathrm{CY}$, Chen YC. Extraction and characterization of fucoidan from six brown macroalgae. J Mar Sci Technol 2016;24:319-28.

15. Yang C, Chung D, Shin IS, Lee H, Kim J, Lee Y, et al. Effects of molecular weight and hydrolysis conditions on anticancer activity of fucoidans from sporophyll of Undaria pinnatifida. Int J Biol Macromol 2008;43:433-7.

16. Hummers WS, Offeman RE. Preparation of graphitic oxide. J Am Chem Soc 1958;80:1339.

17. Marcano DC, Kosynkin DV, Berlin JM, Sinitskii A, Sun Z, Slesarev $A$, et al. Improved synthesis of graphene oxide. ACS Nano 2010;4:4806-14.

18. Bao H, Pan Y, Ping Y, Sahoo NG, Wu T, Li L, et al. Chitosanfunctionalized graphene oxide as a nanocarrier for drug and gene delivery. Small 2011;7:1569-78.
19. Muthukumarasamyvel T, Baskar R, Chandirasekar S, Umamaheswari K, Rajendiran N. Hierarchical self-assembly of bile-acid-derived dicationic amphiphiles and their toxicity assessment on microbial and mammalian systems. ACS Appl Mater Interfaces 2016;8:25111-26.

20. Deb A, Vimala R. Natural and synthetic polymer for graphene oxide mediated anticancer drug delivery-a comparative study. Int J Biol Macromol 2018;107:2320-33.

21. Reshma, Arun KP, Brindha P. In vitro anti-inflammatory, antioxidant and nephroprotective studies on leaves of Aegle marmelos and Ocimum sanctum. Asian J Pharm Clin Res 2014;7:121-9.

22. Dash S, Murthy PN, Nath L, Chowdhury P. Kinetic modeling on drug release from controlled drug delivery systems. Acta Pol Pharm 2010;67:217-23.

23. Gouda R, Baishya H, Qing Z. Application of mathematical models in drug release kinetics of carbidopa and levodopa ER tablets. J Drugs 2017;6:171.

24. Deepachitra R, Chamundeeswari M, Santhosh Kumar B, Krithiga G, Prabu P, Pandima Devi M, et al. Osteo mineralization of fibrin-decorated graphene oxide. Carbon 2013;56:64-76.

25. Mona MK, Hasan T. Development of a graphene oxide/chitosan nanocomposite for the removal of picric acid from aqueous solutions: study of sorption parameters. Colloids Surf B 2017;160:671-81.

26. Martínez Ibarra DM, Sanchez Machado DI, Lopez Cervantes J, Campas Baypoli ON, Sanches Silva A, Madera Santana TJ. Hydrogel wound dressings based on chitosan and xyloglucan: development and characterization. J Appl Polym Sci 2019;136:1-10.

27. Ayoub A, Venditti RA, Pawlak JJ, Salam A, Hubbe MA. Novel hemicellulose-chitosan biosorbent for water desalination and heavy metal removal. ACS Sustain Chem Eng 2013;1:1102-9. 